
\title{
25 Research Soure \\ Effect of BRCA1 and BRCA2 mutations on endometrial carcinoma survival rates
}

\author{
Aifen Wang ( $\square$ aifen@njucm.edu.cn ) \\ Zhangjiagang TCM Hospital Affiliated to Nanjing University of Chinese Medicine \\ https://orcid.org/0000-0002-1868-1890 \\ Robert W Holloway \\ Florida Hospital Orlando \\ Zihan Zhao \\ University of California Santa Barbara \\ Ziyue Zhang \\ University of California San Diego
}

\section{Research article}

Keywords: Endometrial carcinoma, BRCA1, BRCA2, prognosis

Posted Date: April 28th, 2020

DOI: https://doi.org/10.21203/rs.3.rs-24271/v1

License: (1) (1) This work is licensed under a Creative Commons Attribution 4.0 International License. Read Full License 


\section{Abstract}

Background: To evaluate Effect of BRCA1 and BRCA2 mutations on endometrial carcinoma survival rates.

Methods : Data were collected from The Cancer Genome Atlas endometrial cancer database for pathogenic mutations in BRCA1 (58) and BRCA2 (77), coexisting BRCA1 /2 mutations (40), and nonmutations (461). Clinicopathological features and survival rates were compared. Survival time was analyzed using combined data and Cox proportional hazard models, with BRCA1 and BRCA2 as timevarying covariates.

Results: Patients with BRCA1 mutations presented with higher risk disease (endometrioid endometrial carcinoma grade 3 and uterine serous carcinoma) than non-carriers and BRCA2 mutation carriers ( $p=$ 0.005 non carriers vs BRCA1 mutation carriers, $p=0.008$ BRCA1 mutation carriers vs BRCA2 mutation carriers). BRCA1 and BRCA2 mutation carriers tended to have more endometrioid endometrial carcinoma grade 3 than non-carrier group. Overall survival (OS) rates were higher for all patients with BRCA1 and BRCA2 mutations than non-carriers. Patients with BRCA2 mutations had the most favorable progression-free survival (PFS), followed by patients with BRCA1 and BRCA2 co-mutations, and then BRCA1 alteration carriers $(p=0.011)$. BRCA1/2 non-carriers had the worst PFS and OS.

Conclusions: Patients with BRCA1 mutations presented with higher risk disease than non-carriers and BRCA2 mutation carriers. BRCA1 and BRCA2 mutation carriers had more favorable OS and PFS than non- BRCA mutation carriers in patients with endometrioid endometrial carcinoma and uterine serous carcinoma.

\section{Background}

Endometrial cancer is the most common gynecologic malignancy in advanced countries [1]. The incidence of endometrial cancer increased $0.7 \%$ per year from 1999 to 2015, according to data from the National Cancer Institute's Surveillance, Epidemiology and End Results program. The mortality of endometrial cancer grew 1\% per year from 1999 to 2015, based on analyses from the Centers for Disease Control and Prevention [2]. Two types of endometrial cancer are classified by clinicohistological features and genetic characteristics. Type 1 endometrial carcinoma is associated with hyperestrogenism and favorable prognosis $[3,4]$. Histologic features of the cancer tissue are more likely to present endometrial gland-like carcinoma. Genetic features are related to PTEN and POLE $[5,6]$. Type 2 endometrial carcinoma is more aggressive than type 1 and is more likely to be associated with $B R C A 1, B R C A 2$ and $\operatorname{TP53}[5,6]$. Clear cell, serous carcinoma, and mixed carcinoma are classified as type 2 endometrial carcinomas. Germline BRCA1 and BRCA2 are well-known risk factors of both ovarian and breast cancer and are identified in 6-15\% of females with epithelial ovarian carcinoma [7-9]. A multinational cohort study of patients with 11,847 BRCA1 mutations revealed a distinct increased risk of uterine carcinoma (HR 2.65, 95\% Cl 1.69-4.16) [10]. However, a prospective study found that the significantly increased risk 
was associated with $B R C A 1 / 2$ deficiencies in patients taking tamoxifen [11]. Some data identified an increased risk of uterine serous carcinoma in patients with BRCA mutations [12-14]. BRCA1/2 mutations are related to homologous recombination, DNA repair, chromatin remodeling, cell cycle checkpoint surveillance, and transcriptional regulation [15]. The clinicopathological features of $B R C A 1 / 2$ mutations carriers differ from features of non-carriers in epithelial ovarian cancer. Patients with $B R C A 1$ mutations tend to have more aggressive histology [15], higher grade [16], and more advanced stage than noncarriers $[16,17]$. Less data was available in BRCA2 mutated epithelial ovarian cancers and uterine serous carcinomas due to lower prevalence $[15,17,18]$. One small cohort identified that prognosis was similar in $B R C A$ mutated uterine serous carcinoma and non-carriers [17]. Another study found that g $B R C A 1$ and gBRCA2 mutation carriers were associated with favorable 5-year overall survival in epithelial ovarian cancer [19]. Some studies revealed that $B R C A 1$ and $B R C A 2$ mutation carriers had better prognosis than non-carriers $[16,18,20]$, whereas others failed to find differences $[21,22]$. One study reported that $B R C A 2$ mutation carriers have more favorable outcomes than non-carriers [23]. Less data is available for prognosis of patients with endometrial carcinomas based on BRCA1 and BRCA2 mutations status [17].

The mechanism of impact of $B R C A 1 / 2$ mutations on prognosis of cancers is unclear. The literature hypothesizes that favorable prognosis of patients with $B R C A 1 / 2$ mutations may be caused by good response to platinum-based chemotherapy regimens $[18,24]$. This hypothesis is consistent with vitro studies that demonstrated that BRCA1 and BRCA2 mutated cells have a good response to agents, such as platinum-based regimens that induce double-strand DNA breaks [25].

From The Cancer Genome Atlas (TCGA) dataset, we aimed to obtain evidence of the effect of BRCA mutations on prognosis of endometrioid endometrial carcinoma and uterine serous carcinoma. Since limited data are available for patients with endometrial cancer with BRCA1 and BRCA2 mutations, these results may provide evidence on the biology of $B R C A 1 / 2$ mutations, the clinical management of mutation carriers, and the potential impact of clinical trial designs, especially regimens targeting $B R C A 1 / 2$ mutations, such as poly (ADP-ribose)-polymerase (PARP) inhibitors [26].

\section{Methods}

\section{Study Design}

The data was obtained from The Cancer Genome Atlas endometrial cancer database (provisional and Pancancer Atlas), which involved 27 U.S. institutions between 1988 and 2014, and was used for this study because of its large sample size and user-friendly data structure [http://www.cbioportal.org]. All study participants had been diagnosed as endometrial carcinoma. The retrospective cohort was generated from 529 patients from the Pancancer Atlas database and 548 patients from the provisional database. After excluding duplicate patients from both databases $(n=441)$, the final cohort comprised 636 patients. Somatic and germline BRCA1 and BRCA2 mutations were not separated. We obtained clinical, demographic, molecular, and pathology information from these patients. Patients were stratified into four groups according to BRCA1 and BRCA2 mutation status: BRCA1 mutation, BRCA2 mutation, 
coexisting BRCA1 and BRCA2 mutation, and non-carriers. Variables recorded for each case were as follows: histology (endometroid endometrial carcinoma and uterine serous carcinoma included), age at diagnosis ( $\nabla 70,50-69,<50$ years old), mean age at diagnosis, myometrial invasion ( $₫ 50 \%$ or $<50 \%$ myometrial invasion), lymph node involvement, and International Federation of Gynecology and Obstetrics (FIGO) stage (2009 version). Overall survival (OS) was defined as the interval from the date of initial surgical resection to the date of death or last contact. Progression-free survival (PFS) was defined as the interval from the date of diagnosis or surgical resection to the date of recurrence, death, or censored last contact.

\section{Statistical Analysis}

\section{Statistical analysis was performed using Mann-Whitney} tests for continuous variables, and chi-square analysis and Fisher's exact test for categorical variables. The Student's ttest was used to analyze body mass index and age of onset. OS and PFS were analyzed using the Kaplan-Meier method and the log-rank tests starting at the date of diagnosis. Analyses of risk factors were performed using multivariate Cox proportional hazards models. For all tests, statistical significance was less than 0.05 ( $p<0.05$, exact 2-tailed). All calculations were performed using SPSS software (Version 23, IBM, USA).

\section{Results}

The study included 636 endometrial cancer cases with pathogenic mutations in BRCA1 (58), BRCA2 (77), both $B R C A 1$ and $B R C A 2(40)$, and non-carriers (461). Data were available on $100 \%$ for age of onset, $83 \%$ for body mass index, $100 \%$ for tumor stage, $100 \%$ for myometrial invasion, $97 \%$ for lymph node involvement, $95.5 \%$ for overall survival and $93.1 \%$ for progression-free interval. Each patient received appropriate therapy by current National Comprehensive Cancer Network (NCCN) guideline. Patients with $B R C A 1$ mutations presented with higher risk disease (endometrioid grade 3 endometrial carcinoma and uterine serous carcinoma) than non-carrier group and $B R C A 2$ mutation carriers ( $p=0.005$, non-carrier group vs $B R C A 1$ mutation carriers; $p=0.008, B R C A 1$ mutation carriers vs $B R C A 2$ mutation carriers). More patients with $B R C A 1$ and $B R C A 2$ mutation occurred in grade 3 endometrioid endometrial carcinomas than non-carrier group. There were fewer patients with BRCA2 and BRCA1 mutation than non-carrier group in uterine serous carcinomas (Table 1). BRCA1 mutations and BRCA2 mutations carriers were 
associated with having a younger age at the time of diagnosis than noncarriers $(p=0.036, B R C A 1$ mutation carriers vs non carriers; $p=0.001, B R C A 2$ mutation carriers vs non carriers). Compared to patients with $B R C A 1$ mutations, body mass index was higher in non-carriers. No significant differences were identified between $B R C A 1$ and $B R C A 2$ mutation carriers for mean age at diagnosis $(p=0.559)$. We failed to detect any differences in body mass index between $B R C A 2$ mutation carriers and $B R C A$ noncarriers $(p=0.120)$, and between BRCA1 carriers and BRCA2 carriers $(p=0.148)$. No significant differences were found in depth for myometrial invasion or lymph node involvement among $B R C A 1$ mutation, BRCA2 mutation, and BRCA1/2 mutation carriers ( $p>0.05$, Table 1$)$. There were no differences identified in stage among $B R C A$ mutated carriers and non-carriers ( $p>0.05$, Table 1$)$. This study did not include patients with coexisting BRCA1 and BRCA2 mutations because of the limited number of cases. 
Table 1

Clinicopathologic characteristics of endometrial carcinoma based on BRCA mutation status. ${ }^{\mathrm{a}} \mathrm{b}$

\begin{tabular}{|c|c|c|c|c|c|c|}
\hline Variable & $\begin{array}{l}\text { Noncarrier } \\
\text { group } \\
(\mathrm{n}=461)\end{array}$ & $\begin{array}{l}\text { BRCA1 mutation } \\
\text { carriers }(\mathrm{n}=58)\end{array}$ & $\begin{array}{l}\text { BRCA2 mutation } \\
\text { carriers }(\mathrm{n}=77)\end{array}$ & $P^{1}$ & $P^{2}$ & $p^{3}$ \\
\hline \multicolumn{7}{|l|}{ Histology } \\
\hline EEC G1 & $\begin{array}{l}76 / 461 \\
(16.5)\end{array}$ & $5 / 58(8.6)$ & 13/77 (16.9) & & $>0.99$ & 0.205 \\
\hline EEC G2 & $\begin{array}{l}107 / 461 \\
(23.2)\end{array}$ & 7/58 (12.1) & 17/77 (22.1) & $<0.001$ & 0.003 & 0.603 \\
\hline EEC G3 & $\begin{array}{l}139 / 461 \\
(30.1)\end{array}$ & $31 / 58(53.4)$ & $37 / 77$ (48.1) & 0.545 & 0.002 & 0.074 \\
\hline USC & $\begin{array}{l}139 / 461 \\
(30.1)\end{array}$ & 15/58 (25.9) & $10 / 77(12.9)$ & & & \\
\hline High risk ${ }^{c}$ & $\begin{array}{l}278 / 461 \\
(60.3)\end{array}$ & 46/58 (79.3) & $47 / 77$ (61.0) & 0.005 & $>0.99$ & 0.008 \\
\hline \multicolumn{7}{|l|}{ Age } \\
\hline $\begin{array}{l}\text { Mean } \pm \\
\text { SD }\end{array}$ & $\begin{array}{l}64.4 \pm \\
10.7\end{array}$ & $61.1 \pm 12.7$ & $59.9 \pm 12.0$ & 0.036 & 0.001 & 0.559 \\
\hline$>70 y$ & $\begin{array}{l}145 / 460 \\
(31.5)\end{array}$ & 11/56 (19.6) & 13/75 (17.3) & & & \\
\hline $50-70 y$ & $\begin{array}{l}279 / 460 \\
(60.7)\end{array}$ & $37 / 56(66.1)$ & $52 / 75(69.3)$ & & & \\
\hline$<50 y$ & $\begin{array}{l}36 / 460 \\
(7.8)\end{array}$ & $8 / 56(14.3)$ & 10/75 (13.3) & & & \\
\hline Missing & $\begin{array}{l}1 / 461 \\
(0.2)\end{array}$ & 2/58 (3.4) & $2 / 77$ (1.3) & & & \\
\hline
\end{tabular}

\section{Myometrial}

invasion

\begin{tabular}{|c|c|c|c|c|c|c|}
\hline$<50 \%$ & $\begin{array}{l}247 / 452 \\
(54.6)\end{array}$ & $29 / 58(50.0)$ & $44 / 75(58.7)$ & 0.571 & 0.668 & 0.256 \\
\hline$>=50 \%$ & $\begin{array}{l}205 / 452 \\
(45.4)\end{array}$ & $29 / 58(50.0)$ & $31 / 75$ (41.3) & & & \\
\hline Missing & $\begin{array}{l}9 / 461 \\
(2.0)\end{array}$ & 0 & $2 / 77$ (1.3) & & & \\
\hline
\end{tabular}

\section{Lymph}




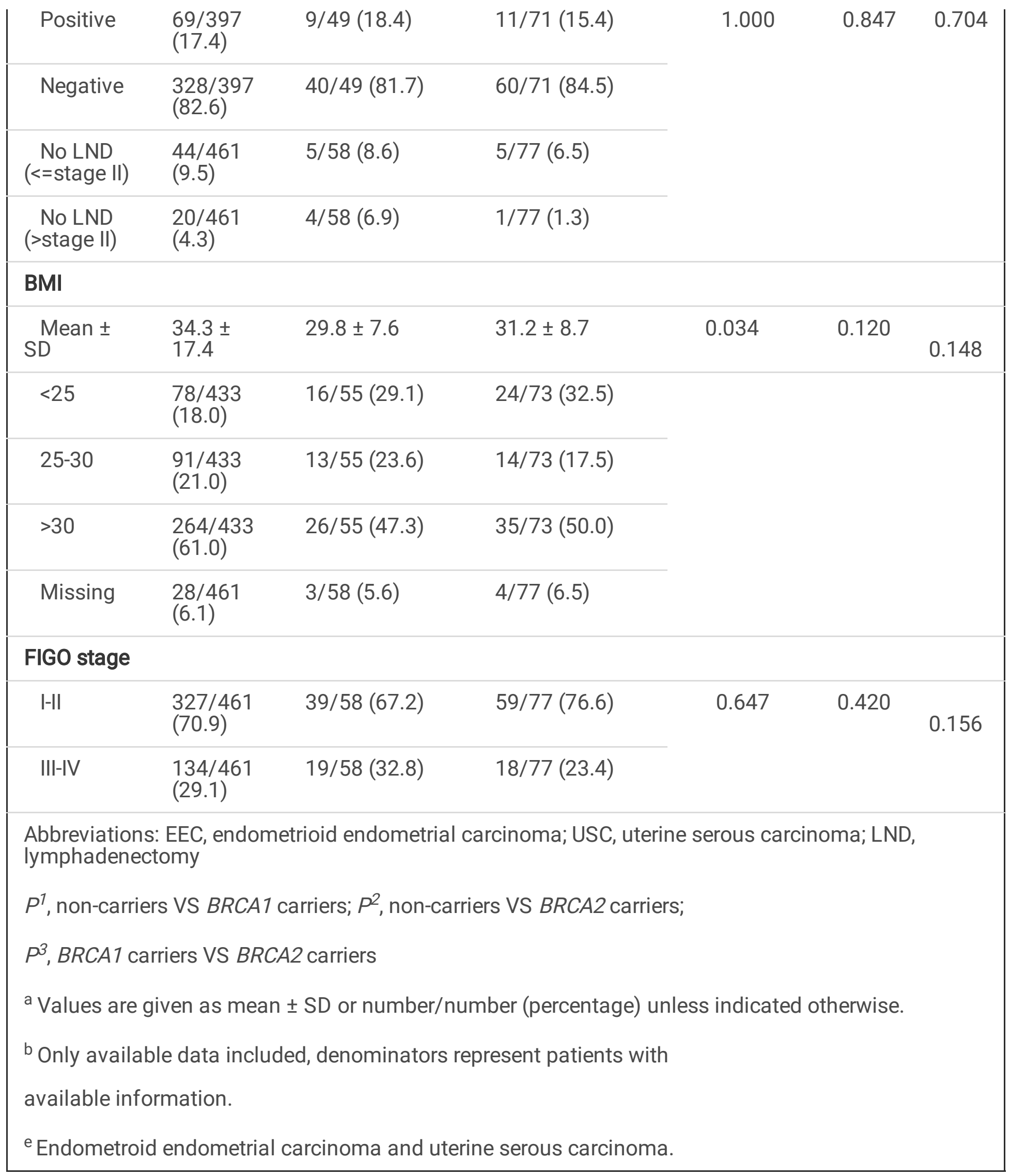

Patients with BRCA1 and/or BRCA2 mutation had more favorable OS ( $p<0.001$, Figure 1$)$ and PFS ( $p=$ 0.008 , Figure 2) than non-carriers (Table 2). Patients with BRCA2 mutations had the longest PFS, 
followed by patients with $B R C A 1 / 2$ mutations, and then $B R C A 1$ mutations. $B R C A 1 / 2$ non-carriers had the worst PFS.

Table 2

Comparison of PFS and OS of patients based on BRCA mutation status

\begin{tabular}{|c|c|c|c|c|}
\hline \multirow[t]{2}{*}{ Variable } & PFS (months) & \multirow[t]{2}{*}{$p$} & OS (months) & \multirow[t]{2}{*}{$p$} \\
\hline & Mean \pm SD $(95 \% \mathrm{Cl})$ & & Mean \pm SD $(95 \% \mathrm{Cl})$ & \\
\hline Noncarriers & $102.8 \pm 3.8(95.3-110.3)$ & \multirow[t]{4}{*}{0.011} & $121.9 \pm 8.6(105.1-138.8)$ & \multirow[t]{4}{*}{$<0.001$} \\
\hline BRCA1 mutation & $191.2 \pm 12.3(167.2-215.3)$ & & $189.9 \pm 21.0(148.9-231.0)$ & \\
\hline$B R C A 2$ mutation & $207.5 \pm 6.9(194.1-221.0)$ & & $180.1 \pm 31.6(118.3-242.0)$ & \\
\hline$B R C A 1 / 2$ mutation & $202.3 \pm 10.8(181.1-223.4)$ & & $176.0 \pm 31.2(114.8-237.1)$ & \\
\hline
\end{tabular}

Independent factors associated with better prognosis in all endometrial adenocarcinomas were $B R C A 1 / 2$ mutation, $<50 \%$ myometrial invasion, and lymph node-negative status (Table 3 ). Compared to noncarriers, the hazard ratio was $0.29(95 \% \mathrm{Cl}, 0.10-0.78 ; \mathrm{p}=0.015)$ for carriers of $B R C A 1,0.17(95 \% \mathrm{Cl}, 0.05-0.54 ; \mathrm{p}=$ $0.003)$ for $B R C A 2$, and $0.29(95 \% \mathrm{Cl}, 0.05-0.59 ; \mathrm{p}=0.037)$ for $B R C A 1 / 2$. We noticed a significant increased risk in ${ }^{3} 50 \%$ myometrial invasion ( $\left.\mathrm{HR}, 2.87 ; 95 \% \mathrm{Cl}, 1.65-5.00 ; p<0.001\right)$. Compared to lymph node-positive status, the hazard ratio of lymph node negative was $0.18(95 \% \mathrm{Cl}, 0.05-0.59 ; p=0.005)$.

Table 3

Relative risk of overall survival (OS) and progression-free interval (PFS) in patients with endometrial carcinoma in relation to histology and grade (Multivariate Cox proportional hazards model).

\begin{tabular}{|c|c|c|c|c|}
\hline \multirow[b]{3}{*}{ Variable name } & \multicolumn{4}{|c|}{ All patients $(N=565)$} \\
\hline & \multicolumn{2}{|c|}{ OS $(414 / 565)$} & \multicolumn{2}{|c|}{ PFS (396/565) } \\
\hline & $\mathrm{HR}[95 \% \mathrm{Cl}]$ & $P$ & $\mathrm{HR}[95 \% \mathrm{Cl}]$ & $\mathrm{P}$ \\
\hline Myometrial invasion ${ }^{3} 50 \%$ vs. $<50 \%$ & $\begin{array}{l}2.87[1.65- \\
5.00]^{-1}\end{array}$ & $<0.001$ & $\begin{array}{l}2.60[1.23- \\
5.38]\end{array}$ & 0.010 \\
\hline $\begin{array}{l}B R C A 1 / 2 \text { mutation vs. } B R C A \\
\text { noncarriers }\end{array}$ & $\begin{array}{l}0.29[0.09- \\
0.93]\end{array}$ & 0.037 & N/A & N/A \\
\hline$B R C A 1$ mutation vs. $B R C A$ noncarriers & $\begin{array}{l}0.29[0.10- \\
0.78]\end{array}$ & 0.015 & N/A & N/A \\
\hline$B R C A 2$ mutation vs. $B R C A$ noncarriers & $\begin{array}{l}0.17[0.05- \\
0.54]\end{array}$ & 0.003 & N/A & $\mathrm{N} / \mathrm{A}$ \\
\hline Lymph node - vs. lymph node + & $\begin{array}{l}0.18[0.05- \\
0.60]\end{array}$ & 0.005 & N/A & N/A \\
\hline
\end{tabular}




\section{Discussion}

To the best of our knowledge, this study was the first to investigate the effect of $B R C A$ mutations on the prognosis of endometrioid endometrial carcinoma. Patients with BRCA1 mutations presented with higher risk disease than non-carrier group and BRCA2 mutation carriers [17]. Among endometrioid endometrial carcinoma group, there were more patients with $B R C A 1$ or $B R C A 2$ mutation than non-carrier group in our study. Patients with $B R C A 1$ and $B R C A 2$ mutations had more favorable outcomes than noncarriers in endometrial carcinoma, which differ from the results of previous studies. There were no significant differences identified in prognosis of uterine serous carcinoma between BRCA mutation carriers and noncarriers [17]. In addition, our findings differ from the analysis of ovarian cancer data from the Cancer Genome Atlas project, and another study that found that patients with BRCA2 mutation had more favorable prognosis than $B R C A 2$ non-carriers $[19,23]$. The survival advantage of patients with $B R C A 1 / 2$ could be associated with intrinsic biological differences, response to therapeutic regimes, or both. The hazard ratios (HR) compared to non-carriers were 0.29 for $B R C A 1$ mutation carriers, 0.17 for $B R C A 2$ mutation carriers, and 0.29 for BRCA1/2 mutation carriers. These findings differ from the hazard ratios for $B R C A 1$ carriers compared to non-carriers reported by Yang et al. (multivariate adjusted HR= 0.76 ) and other analysis (multivariate adjusted $\mathrm{HR}=0.73$ ) among patients with ovarian cancer $[19,23]$. The reason for these differences could be that we did not separate germline and somatic BRCA mutations in this study. The survival differences between $B R C A 2$ mutation carriers and non-carriers are attenuated and may even be reversed for $B R C A 1$ mutation carriers in the long run $[27,28]$.

We presume that resistance to platinum-based treatment was more likely to occur in endometrial carcinoma with BRCA2 mutations than with BRCA1 mutations, leading to reverse prognosis. The mechanism of resistance to platinum-based treatment in BRCA1 and BRCA2 carriers was associated with homologous recombination restoration that occurs by inactivation of the p53-binding protein1 (53BP1), which is important in maintaining the balance between homologous recombination and non-homologous end joining, and is transferred to non-homologous end joining in BRCA7-mutant cells [29]. One study reported that $B R C A 1 / 2$ mutations are associated with favorable prognosis in short-term surveillance, but this advantage attenuates over time, and homologous recombination was reversed through induction by platinum-based treatment in BRCA1 carriers [27]. Newer agents, especially those effective in $B R C A 1 / 2$ mutation carriers, like PARP inhibitors, are required for treatment of both primary and relapsed cancers with $B R C A 1 / 2$ mutations and should be investigated in clinical trials for analysis of long-term survival.

We saw no differences in lymph node involvement among BRCA1-or BRCA2-mutated tumors and noncarriers in this study. These results differed from ovarian cancer, in which significant differences were found between patients with $B R C A$ mutations and non-carriers; however, BRCA1-and BRCA2-related tumors were similar to each other [19]. Our findings contrast with results of breast cancer, in which substantial differences were revealed between $B R C A 1$ - and BRCA2-associated disease [30, 31]. We noticed higher grade histology in endometrial cancers with $B R C A 1$ and $B R C A 2$ alterations, which is similar to the result of ovarian cancer $[15,16]$. Our results revealed that fewer $B R C A 2$ and $B R C A 1$ mutations occurred in uterine serous carcinomas than non-carrier group. BRCA1 mutations occurred more 
frequently than non-mutations in higher risk disease (endometroid endometrial carcinomas and uterine serous carcinomas). Some data showed an increased risk of uterine serous carcinoma in patients with BRCA mutation [12-14]. BRCA1 carriers and non-carriers presented no significant differences in advanced disease in our study, which is similar to the result of previous studies [17]. However, less data is available for BRCA2-mutated epithelial ovarian cancer or endometrial carcinoma due to a lower prevalence $[15,18]$.

Data showed that women with $B R C A$ mutated recurrent or advanced ovarian cancer responded to olaparib if they failed multiple prior lines of chemotherapy [32, 33]. PARP inhibitors, such as niraparib, rucaparib, and olaparib, are Food and Drug Administration-approved anti-cancer drugs administered after failure of two or three prior lines of treatment in patients with BRCA-mutated recurrent ovarian cancer. For now, there are no clinical trials available for endometrial carcinoma with BRCA mutations using PARP inhibitors. The findings of our study may suggest potential management of patients with BRCA-mutated endometrial carcinoma. Patients could receive individual management if they were stratified based on $B R C A$ mutation status. In addition, our study revealed that BRCA1- and/or BRCA2-mutated endometrial carcinomas had better prognosis than non-carriers, even in high-grade or advanced-stage disease. In the future, our findings could be used for counseling patients on their prognosis.

This study had several limitations, including its retrospective nature and small sample size. Some clinical information, such as family history, was missing. Further studies are necessary to identify differences between prognosis of endometrial carcinomas and germline or somatic BRCA mutations in larger cohorts.

\section{Conclusions}

Patients with $B R C A 1$ mutations presented with higher risk endometrial carcinoma than non-carrier group or $B R C A 2$ mutation carriers. BRCA1 or BRCA2 mutation carriers tended to have more grade 3 endometrioid endometrial carcinoma than non-carrier group. BRCA1 and BRCA2 mutation carriers have more favorable overall survival and progression-free survival among patients with endometrial carcinoma. Independent factors associated with more favorable prognosis for all endometrial adenocarcinomas included $B R C A 1 / 2$ mutation, $<50 \%$ myometrial invasion, and negative lymph node status.

\section{Abbreviations}

FIGO: International Federation of Gynecology and Obstetrics

OS: Overall survival

PFS: Progression-free survival

PARP: poly (ADP-ribose)-polymerase 


\section{Declatations}

\section{Authors contributions}

AW analyzed and interpreted the data and was a major contributor in writing the manuscript; $\mathrm{AW}$ and $\mathrm{RH}$ interpreted the data; ZZ and ZZ abstracted the data; AW designed the work; AW designed the work and interpreted the data. All authors read and approved the final manuscript.

\section{Acknowledgement}

We thank International Science Editing (http://www.internationalscienceediting.com) for editing this manuscript.

\section{Ethics approval and consent to participate}

The study was approved by the Ethics Committee of Zhangjiagang Hospital TCM Affiliated to Nanjing University of Chinese Medicine (committee's reference number: 2018-1071). Because all patients involved in TCGA were publicly published. The Ethics Committee approved this consent procedure.

\section{Availability of data and materials}

All data and materials were from TCGA which was published publicly.

\section{Consent for publication}

Not applicable.

\section{Competing interests}

The authors declare that they have no competing interests.

\section{References}

[1] Siegel RL, Miller KD, Jemal A. Cancer statistics, 2020. CA Cancer J Clin.2020;70(1)7-30. doi10.3322/caac. 21590 .

[2] Kuehn B. Uterine Cancer Concern. JAMA.2019;321(4)336. doi10.1001/jama.2018.21059. 
[3] Furness S, Roberts H, Marjoribanks J, Lethaby A, Hickey M, Farquhar C. Hormone therapy in postmenopausal women and risk of endometrial hyperplasia. Cochrane Database Syst Rev.2009(2)CD000402. doi10.1002/14651858.CD000402.pub3.

[4] Karageorgi S, Hankinson SE, Kraft P, De Vivo I. Reproductive factors and postmenopausal hormone use in relation to endometrial cancer risk in the Nurses' Health Study cohort 1976-2004. Int J Cancer.2010;126(1)208-216. doi10.1002/ijc.24672.

[5] Jasin M. Homologous repair of DNA damage and tumorigenesis: the BRCA connection. Oncogene.2002;21(58)8981-8993. doi10.1038/sj.onc.1206176.

[6] Lavie O, Ben-Arie A, Segev Y, Faro J, Barak F, Haya N, et al. BRCA germline mutations in women with uterine serous carcinoma-still a debate. Int J Gynecol Cancer.2010;20(9)1531-1534. doi10.1111/IGC.0b013e3181cd242f.

[7] Zhang S, Royer R, Li S, McLaughlin JR, Rosen B, Risch HA, et al. Frequencies of BRCA1 and BRCA2 mutations among 1,342 unselected patients with invasive ovarian cancer. Gynecol Oncol.2011;121(2)353-357. doi10.1016/j.ygyno.2011.01.020.

[8] Pal T, Permuth-Wey J, Betts JA, Krischer JP, Fiorica J, Arango H, et al. BRCA1 and BRCA2 mutations account for a large proportion of ovarian carcinoma cases. Cancer.2005;104(12)2807-2816. doi10.1002/cncr.21536.

[9] O'Donovan PJ, Livingston DM. BRCA1 and BRCA2: breast/ovarian cancer susceptibility gene products and participants in DNA double-strand break repair. Carcinogenesis.2010;31(6)961-967. doi10.1093/carcin/bgq069.

[10] Thompson D, Easton DF, Breast Cancer Linkage C. Cancer Incidence in BRCA1 mutation carriers. J Natl Cancer Inst.2002;94(18)1358-1365.

[11] Beiner ME, Finch A, Rosen B, Lubinski J, Moller P, Ghadirian P, et al. The risk of endometrial cancer in women with BRCA1 and BRCA2 mutations. A prospective study. Gynecol Oncol.2007;104(1)7-10. doi10.1016/j.ygyno.2006.08.004.

[12] Biron-Shental T, Drucker L, Altaras M, Bernheim J, Fishman A. High incidence of BRCA1-2 germline mutations, previous breast cancer and familial cancer history in Jewish patients with uterine serous papillary carcinoma. Eur J Surg Oncol.2006;32(10)1097-1100. doi10.1016/j.ejso.2006.03.032.

[13] Hornreich G, Beller U, Lavie O, Renbaum P, Cohen Y, Levy-Lahad E. Is uterine serous papillary carcinoma a BRCA1-related disease? Case report and review of the literature. Gynecol Oncol.1999;75(2)300-304. doi10.1006/gyno.1999.5568.

[14] Lavie O, Hornreich G, Ben-Arie A, Rennert G, Cohen Y, Keidar R, et al. BRCA germline mutations in Jewish women with uterine serous papillary carcinoma. Gynecol Oncol.2004;92(2)521-524. 
doi10.1016/j.ygyno.2003.11.009.

[15] Lakhani SR, Manek S, Penault-Llorca F, Flanagan A, Arnout L, Merrett S, et al. Pathology of ovarian cancers in BRCA1 and BRCA2 carriers. Clin Cancer Res.2004;10(7)2473-2481. doi10.1158/1078-0432.ccr1029-3.

[16] Chetrit A, Hirsh-Yechezkel G, Ben-David Y, Lubin F, Friedman E, Sadetzki S. Effect of BRCA1/2 mutations on long-term survival of patients with invasive ovarian cancer: the national Israeli study of ovarian cancer. J Clin Oncol.2008;26(1)20-25. doi10.1200/JC0.2007.11.6905.

[17] Kadan Y, Raviv O, Segev Y, Lavie O, Bruchim I, Fishman A, et al. Impact of BRCA mutations on outcomes among patients with serous endometrial cancer. Int J Gynaecol Obstet.2018;142(1)91-96. doi10.1002/ijgo.12486.

[18] Cass I, Baldwin RL, Varkey T, Moslehi R, Narod SA, Karlan BY. Improved survival in women with BRCAassociated ovarian carcinoma. Cancer.2003;97(9)2187-2195. doi10.1002/cncr.11310.

[19] Bolton KL, Chenevix-Trench G, Goh C, Sadetzki S, Ramus SJ, Karlan BY, et al. Association between BRCA1 and BRCA2 mutations and survival in women with invasive epithelial ovarian cancer. JAMA.2012;307(4)382-390. doi10.1001/jama.2012.20.

[20] Boyd J, Sonoda Y, Federici MG, Bogomolniy F, Rhei E, Maresco DL, et al. Clinicopathologic features of BRCA-linked and sporadic ovarian cancer. JAMA.2000;283(17)2260-2265.

[21] Buller RE, Shahin MS, Geisler JP, Zogg M, De Young BR, Davis CS. Failure of BRCA1 dysfunction to alter ovarian cancer survival. Clin Cancer Res.2002;8(5)1196-1202.

[22] Pharoah PD, Easton DF, Stockton DL, Gayther S, Ponder BA. Survival in familial, BRCA1-associated, and BRCA2-associated epithelial ovarian cancer. United Kingdom Coordinating Committee for Cancer Research (UKCCCR) Familial Ovarian Cancer Study Group. Cancer Res.1999;59(4)868-871.

[23] Yang D, Khan S, Sun Y, Hess K, Shmulevich I, Sood AK, et al. Association of BRCA1 and BRCA2 mutations with survival, chemotherapy sensitivity, and gene mutator phenotype in patients with ovarian cancer. JAMA.2011;306(14)1557-1565. doi10.1001/jama.2011.1456.

[24] Tan DS, Rothermundt C, Thomas K, Bancroft E, Eeles R, Shanley S, et al. "BRCAness" syndrome in ovarian cancer: a case-control study describing the clinical features and outcome of patients with epithelial ovarian cancer associated with BRCA1 and BRCA2 mutations. J Clin Oncol.2008;26(34)55305536. doi10.1200/JCO.2008.16.1703.

[25] Foulkes WD. BRCA1 and BRCA2: chemosensitivity, treatment outcomes and prognosis. Fam Cancer.2006;5(2)135-142. doi10.1007/s10689-005-2832-5. 
[26] Audeh MW, Carmichael J, Penson RT, Friedlander M, Powell B, Bell-McGuinn KM, et al. Oral poly(ADPribose) polymerase inhibitor olaparib in patients with BRCA1 or BRCA2 mutations and recurrent ovarian cancer: a proof-of-concept trial. Lancet.2010;376(9737)245-251. doi10.1016/S0140-6736(10)60893-8.

[27] Candido-dos-Reis FJ, Song H, Goode EL, Cunningham JM, Fridley BL, Larson MC, et al. Germline mutation in BRCA1 or BRCA2 and ten-year survival for women diagnosed with epithelial ovarian cancer. Clin Cancer Res.2015;21(3)652-657. doi10.1158/1078-0432.CCR-14-2497.

[28] McLaughlin JR, Rosen B, Moody J, Pal T, Fan I, Shaw PA, et al. Long-term ovarian cancer survival associated with mutation in BRCA1 or BRCA2. J Natl Cancer Inst.2013;105(2)141-148. doi10.1093/jnci/djs494.

[29] E G, S R, Jonker. Resistence to PARP inhibitors: lessons from preclinical models of BRCA-associated cancer. Annu Rev Cancer Biol.2019.

[30] Lakhani SR, Van De Vijver MJ, Jacquemier J, Anderson TJ, Osin PP, McGuffog L, et al. The pathology of familial breast cancer: predictive value of immunohistochemical markers estrogen receptor, progesterone receptor, HER-2, and p53 in patients with mutations in BRCA1 and BRCA2. J Clin Oncol.2002;20(9)2310-2318. doi10.1200/JC0.2002.09.023.

[31] Lakhani SR, Jacquemier J, Sloane JP, Gusterson BA, Anderson TJ, van de Vijver MJ, et al. Multifactorial analysis of differences between sporadic breast cancers and cancers involving BRCA1 and BRCA2 mutations. J Natl Cancer Inst.1998;90(15)1138-1145.

[32] Kaufman B, Shapira-Frommer R, Schmutzler RK, Audeh MW, Friedlander M, Balmana J, et al. Olaparib monotherapy in patients with advanced cancer and a germline BRCA1/2 mutation. J Clin Oncol.2015;33(3)244-250. doi10.1200/JC0.2014.56.2728.

[33] Matulonis UA, Penson RT, Domchek SM, Kaufman B, Shapira-Frommer R, Audeh MW, et al. Olaparib monotherapy in patients with advanced relapsed ovarian cancer and a germline BRCA1/2 mutation: a multistudy analysis of response rates and safety. Ann Oncol.2016;27(6)1013-1019. doi10.1093/annonc/mdw133.

\section{Figures}




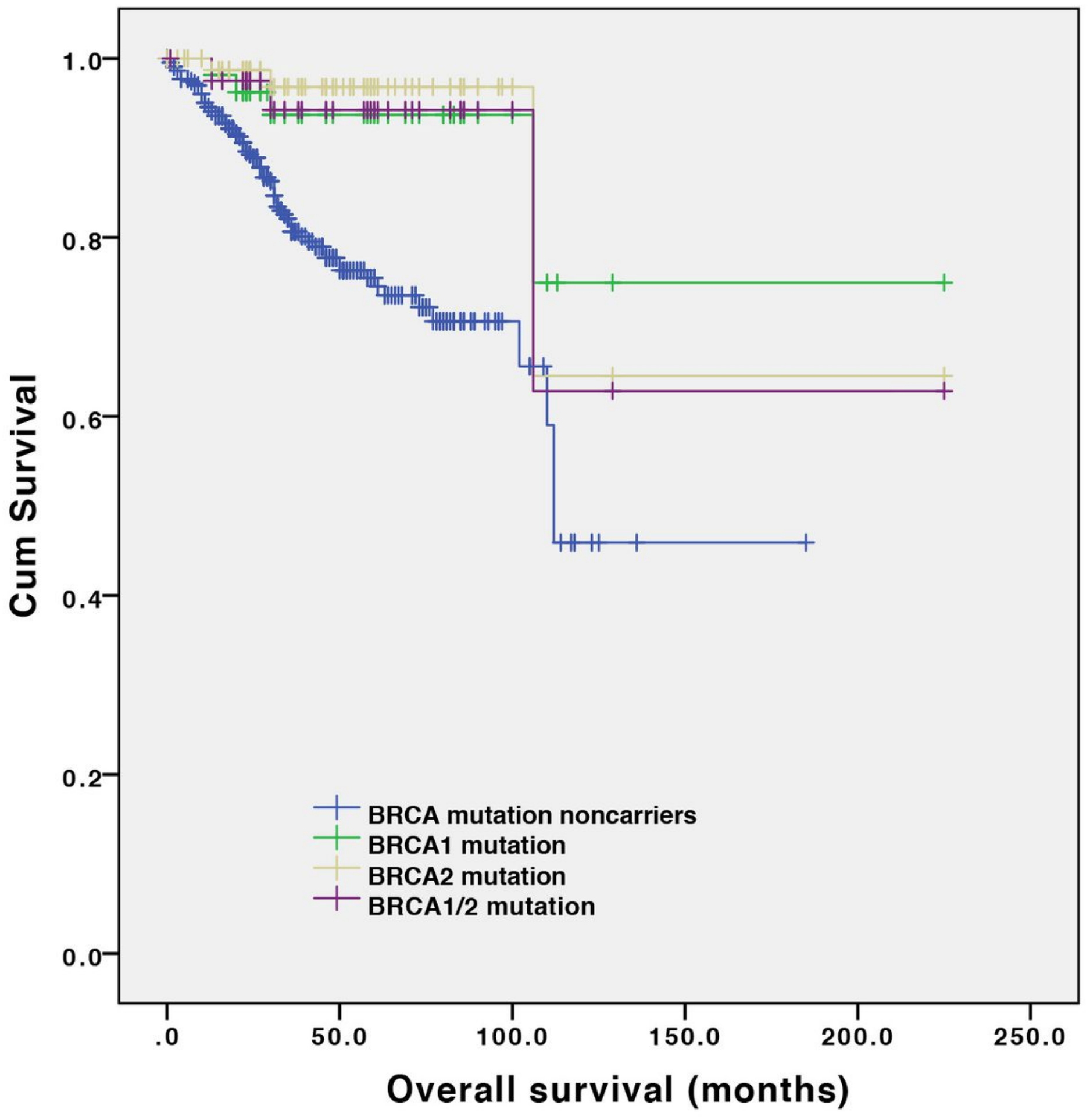

Figure 1

Overall survival from endometrial carcinoma based on BRCA mutation status Legend: Overall survival (OS) rates were higher for all patients with BRCA1 or BRCA2 mutations than non-carriers. 


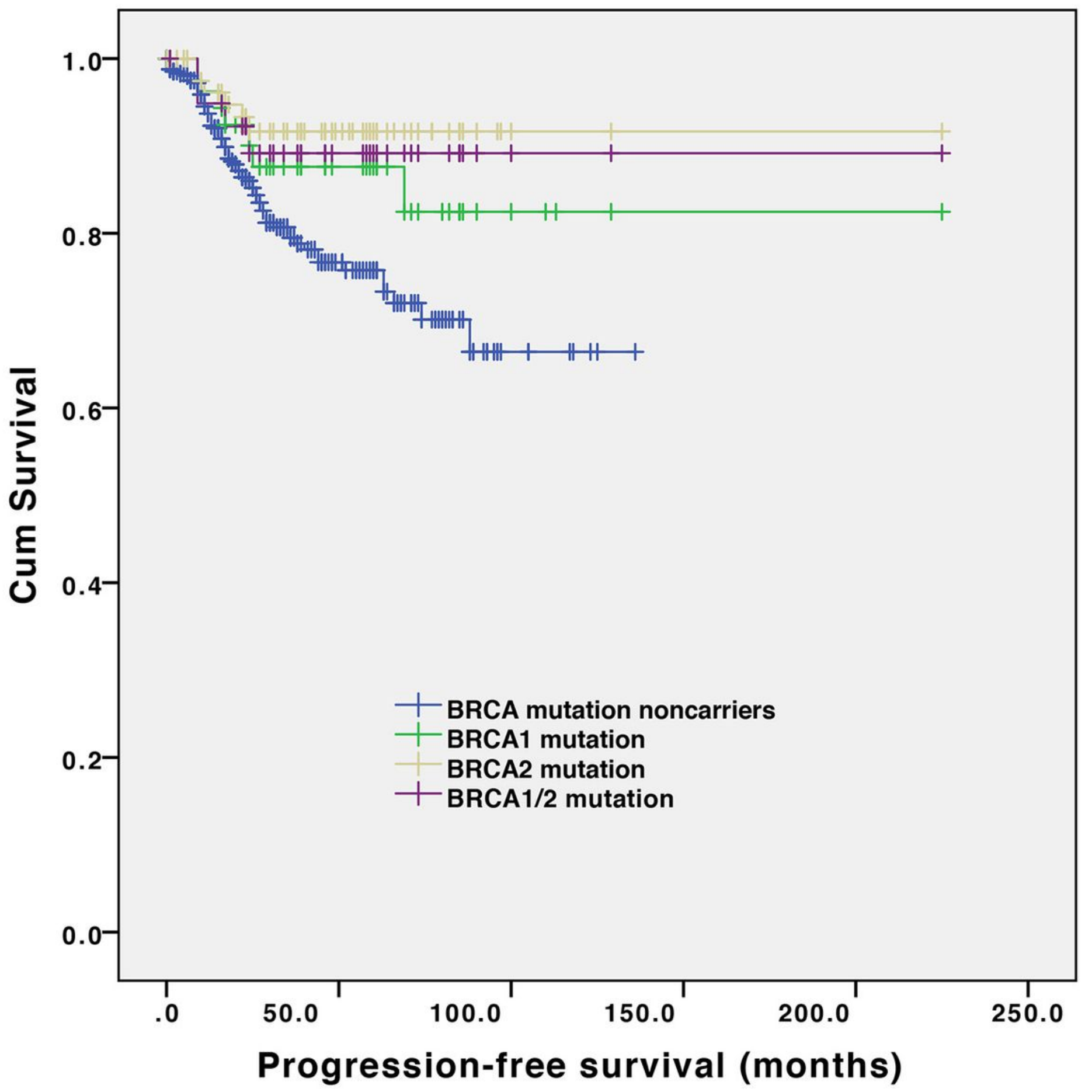

Figure 2

Progression-free survival from endometrial carcinoma based on BRCA mutation status Legend: Patients with BRCA2 mutations had the most favorable progression-free survival (PFS), followed by patients with BRCA1 and BRCA2 co-mutations and BRCA1 alteration carriers. BRCA1/2 non-carriers had the worst PFS. 